\title{
Superficial spreading melanoma in a thermal burn
}

\section{scar}

\section{Fatima Zahra Elfatoiki', Ghita Sami', Hayat Dahbi Skali', Fouzia Hali', Mounia Diouri², Farida Marnissi $^{3}$, Soumia Chiheb ${ }^{1}$}

${ }^{1}$ Department of Dermatology, Ibn Rochd UHC, 1 quartier des Hopitaux 20000, Casablanca, Morocco, ${ }^{2}$ Department of plastic surgery. Ibn Rochd UHC, 1 quartier des Hopitaux 20000, Casablanca, Morocco, ${ }^{3}$ Department of Anatomo-Pathology, Ibn Rochd UHC, 1 quartier des Hopitaux 20000, Casablanca, Morocco

Corresponding author: Dr. Fatima Zahra Elfatoiki, E-mail: Fatiza59@hotmail.fr

\begin{abstract}
Cases of melanoma on thermal burn scars have exceptional been reported. We report a case of superficial spreading melanoma arising after about 30 years after a burn injury. 54 year-old women presented with pigmented lesion that emerged on her old burn scar of the back. The cutaneous biopsy showed a melanoma. There was no palpable regional adenopathy or any distant metastases detectable. An excision with a $2 \mathrm{~cm}$ margin was performed. We would like to highlight the importance of earlier treatment of necrotic burn scar with very long follow-up.
\end{abstract}

Key words: Melanoma; Scar; Burn; Back; Surgery

How to cite this article: Elfatoiki FZ, Sami G, Dahbi Skali H, Hali F, Diouri M, Marnissi F, Chiheb S. Superficial spreading melanoma in a thermal burn scar. Our Dermatol Online. 2020;11(e):e83.1-e83.4.

Submission: 14.10.2019; Acceptance: 05.01.2020

DOI: 10.7241 /ourd.2020e.83 


\section{Mélanome à extension superficielle sur cicatrice de brulure thermique}

\section{Fatima Zahra Elfatoiki ${ }^{1}$, Ghita Sami', Hayat Dahbi Skali', Fouzia Hali', Mounia Diouri², Farida Marnissi $^{3}$, Soumia Chiheb ${ }^{1}$}

${ }^{1}$ Department of Dermatology, Ibn Rochd UHC, 1 quartier des Hopitaux 20000, Casablanca, Morocco, ${ }^{2}$ Department of plastic surgery. Ibn Rochd UHC, 1 quartier des Hopitaux 20000, Casablanca, Morocco, ${ }^{3}$ Department of Anatomo-Pathology, Ibn Rochd UHC, 1 quartier des Hopitaux 20000, Casablanca, Morocco

Corresponding author: Dr. Fatima Zahra Elfatoiki, E-mail: Fatiza59@hotmail.fr

\section{RÉSUMÉ}

Les mélanomes sur cicatrices de brulures sont exceptionnels. Nous rapportons le cas d'un mélanome sur cicatrice de brulure thermique avec un délai d'apparition de 30 ans après la brulure. Patiente de 54 ans, s'est présentée pour une lésion pigmentée apparue sur sa cicatrice ancienne de brulure du dos. La biopsie cutanée était en faveur de mélanome. Il n'a pas été noté d'adénopathies palpables ou de métastases à distance. Une exérèse chirurgicale était réalisée avec des marges de $2 \mathrm{~cm}$. Nous soulignons l'importance de l'excision chirurgicale précoce des cicatrices nécrotiques de brulures et du suivi régulier des cicatrices de brulures chroniques.

Mots Clé: Mélanome; Cicatrice; Brulure; Dos; Chirurgie

\section{INTRODUCTION}

La transformation maligne est une complication commune des cicatrices de brulure. Le carcinome épidermoïde est le cancer le plus fréquent sur ce type de cicatrices. La survenue de mélanome sur cicatrice de brulure est exceptionnelle. Nous rapportons le cas d'un mélanome à extension superficielle sur cicatrice de brulure thermique avec un délai d'apparition de 30 ans après la brulure.

\section{OBSERVATION}

Patiente de 54 ans avec un antécédent de brulure thermique de 2ème degré du dos il y a 30 ans, a vu apparaitre depuis 6 mois une lésion pigmentée au sein de la cicatrice de brulure. Lexamen clinique retrouvait au niveau du dos, une large cicatrice de brulure du dos rétractile en périphérie, scléro-atrophique et hypohromique au centre, surmontée au centre d'une pigmentation inhomogène mal limitée à bordure irrégulière de $8 \mathrm{~cm}$ de grand axe, avec quelques lésions pigmentaires satellites (Figs. 1 et 2). Lexamen dermoscopique avait révélé une pigmentation irrégulière inhomogène polychromatique, avec la présence de voile gris bleu par endroit, en faveur d'un mélanome. L'examen des aires ganglionnaires était sans particularité. Le reste de l'examen clinique était normal. La biopsie cutanée de la lésion pigmentée avait montré une prolifération tumorale mélanocytaire en faveur d'un mélanome. Le scanner thoraco-abdomino-pelvien était sans particularité. L'échographie des aires ganglionnaires axillaires et inguinales était normale. Une exérèse chirurgicale était réalisée avec des marges de $2 \mathrm{~cm}$ suivie d'une fermeture par suture directe sans décollement. Lexamen histologique et immunohistochimique de la pièce opératoire était en faveur d'un mélanome à extension superficielle (SSM) pauvre en mitoses sans ulcération avec des signes de régression et un indice de Breslow de 1,2 mm (Figs. 3 et 4). Les limites d'exérèse profonde et latérales étaient saines. La tomographie par émission de positrons n'avait pas montré de localisations secondaires.

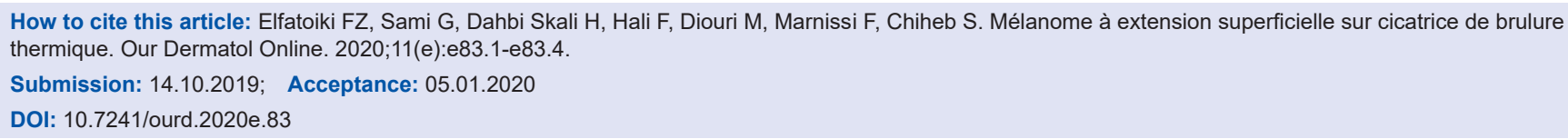




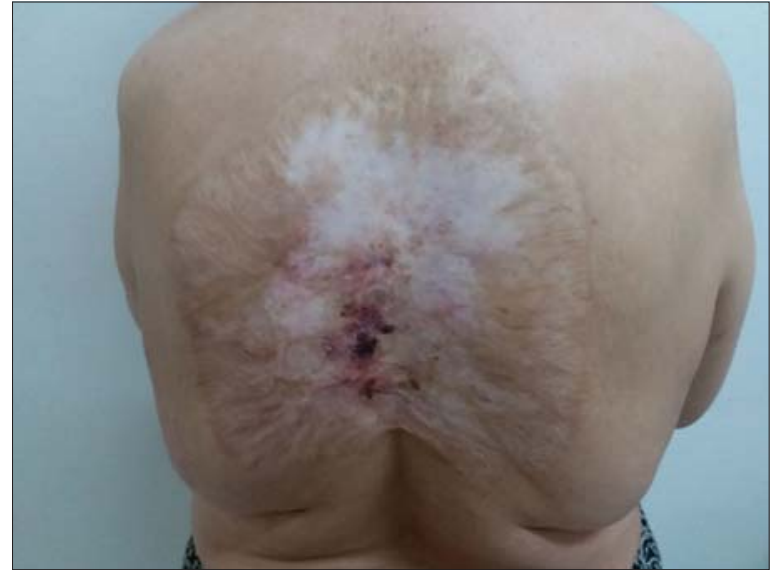

Figura 1: Large burn scar of the back, sclero-atrophic and hypohromic in the center, surmounted at the center of an inhomogeneous pigmentation with irregular border.

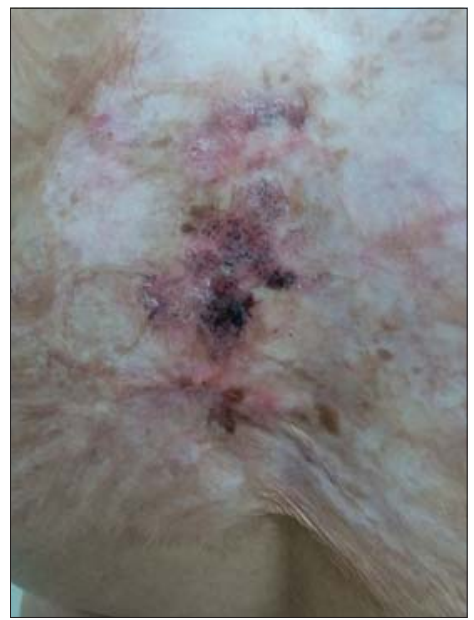

Figura 2: Large burn scar of the back, sclero-atrophic and hypohromic in the center, surmounted at the center of an inhomogeneous pigmentation with irregular border.

\section{DISCUSSION}

La transformation maligne est une complication commune des cicatrices de brulures. En effet, depuis la description originale de Marjolin au 19ème siècle, la notion de transformation maligne de cicatrices de brulures est devenue classique. Environ 2\% de cicatrices de brulures peuvent se compliquer de dégénérescence maligne plusieurs années après la survenue de la brûlure [1]. Le carcinome épidermoïde constitue le cancer des cicatrices de brulure chronique le plus fréquent, suivi par le carcinome basocellulaire. La survenue de mélanome sur cicatrice de brulure est exceptionnelle [1].

La pathogénie de la survenue des néoplasie dont le mélanome sur cicatrices de brulures chroniques n'est pas totalement élucidée. Certaines hypothèses

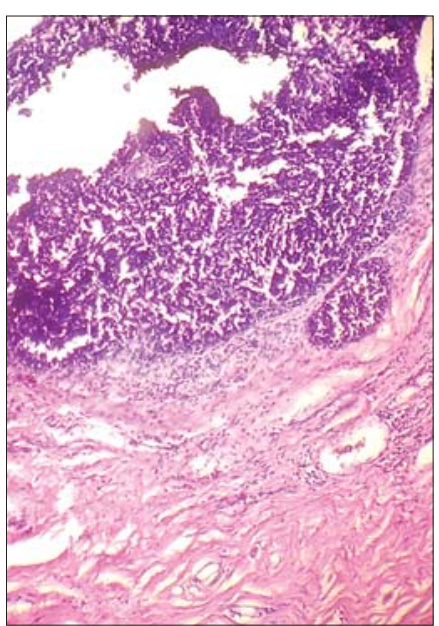

Figura 3: Lentigenous melanocytic tumoral proliferation of the epidermis with infiltration of the papillary dermis.

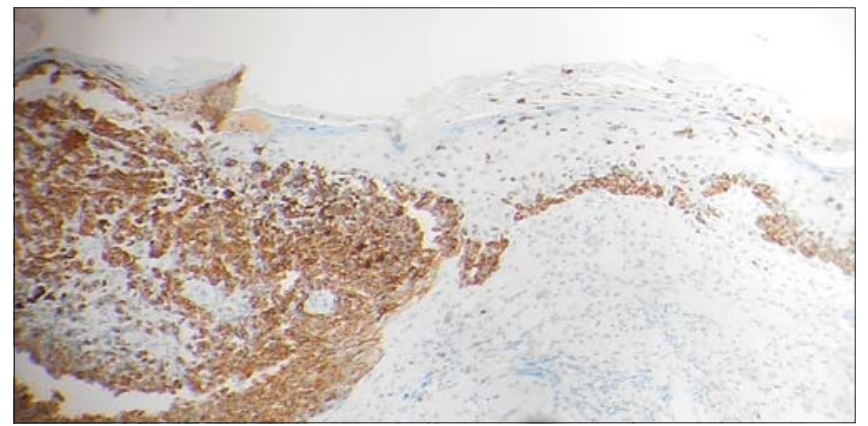

Figura 4: Expression of the melanin marker HMB-45 by the tumoral cells.

avancent la possibilité de synthèse de toxines carcinogènes au niveau du site de brulure. D'autres soulignent l'environnement cicatriciel favorisant de l'apparition des néoplasies. Par contre, l'exposition solaire ne semble pas avoir un rôle dans la pathogénie de ces néoplasies puisque les brulures prédominent sur des zones presque constamment photoprotégées [1].

Dans une revue de littérature réalisée par Ribero et al. ayant inclus 38 cas de mélanomes sur cicatrices chroniques de brulure, l'âge moyen des patients à la survenue de la brulure était de 13,5 ans alors que l'âge moyen des patients au diagnostic du mélanome était de 55,8 ans. La période de latence après la survenue de la brulure peut varier de 0 à 71 ans avec une moyenne de 43,8 ans [2]. Notre patiente était âgée de 54 ans au diagnostic du mélanome avec un délai de 30 ans après la survenue de la brulure.

Dans la même étude de Ribero et al., la tumeur est le plus souvent nodulaire ou ulcérée, la forme primitive multiple est possible. La pigmentation est fréquente, 
mais des mélanomes achromiques sont possibles. Les mélanomes étaient localisés préférentiellement au niveau des extrémités suivies par le tronc et le dos. Lindice de Breslow variait entre 0,4 et $15 \mathrm{~mm}$ avec une moyenne de 5,07 mm [3]. Dans notre cas, le mélanome était pigmenté, de type SSM avec une localisation au niveau du dos et un Breslow de 1,2 mm.

Le traitement du mélanome sur cicatrice de brulure est essentiellement chirurgical. Son évolution rejoint celle du mélanome classique. La progression est en général rapide avec possibles localisations secondaires $[2,3]$. Dans notre cas, le traitement consistait en une exérèse large de la tumeur suivie d'une fermeture par suture directe. Le bilan d'extension initiale n'avait pas montré de localisations secondaires ganglionnaires ou viscérales.

À la vue de notre observation, nous voulons insister sur l'intérêt de la prise en charge initiale adéquate de toute brulure importante et de la surveillance régulière des cicatrices de brulure. En effet, nous soulignons l'importance de l'excision chirurgicale précoce des cicatrices nécrotiques de brulures afin d'améliorer l'état local et de réduire ainsi le risque de la transformation maligne. D'autre part, le suivi régulier des cicatrices chroniques de brulure va permettre le diagnostic et la prise en charge précoces de la néoplasie afin de limiter le risque de dissémination.

\section{Statement of Informed Consent}

The examination of the patient was conducted according to the Declaration of Helsinki principles.

The authors certify that they have obtained all appropriate patient consent forms. In the form the patient(s) has/have given his/her/their consent for his/her/their images and other clinical information to be reported in the journal. The patients understand that their names and initials will not be published and due efforts will be made to conceal their identity, but anonymity cannot be guaranteed.

\section{RÉFÉRENCES}

1. Cantwell P, Brooks A. Multiple melanoma in a burns scar. BMJ Case Rep. 2018;11:e227295.

2. Ribero S, Grassi M, Caliendo V, Lauro D, Macripò G. Melanoma arising on a scar 10 years after a burn: management and review of the literature. Ann Plast Surg. 2012;69:27-9.

3. Lee HB, Han SE, Chang LS, Lee SH. Malignant melanoma on a thermal burn scar. Arch Craniofac Surg. 2019;20:58-61.

Copyright by Fatima Zahra Elfatoiki, et al. This is an open-access article distributed under the terms of the Creative Commons Attribution License, which permits unrestricted use, distribution, and reproduction in any medium, provided the original author and source are credited.

Source of Support: Nil, Conflict of Interest: None declared. 